\title{
Janus a god with two faces: death and survival utilise same mechanisms conserved by evolution
}

\author{
P Nicotera $^{*, 1}, \mathrm{OH}$ Petersen ${ }^{2}, \mathrm{G} \mathrm{Melino}^{1,3}$ and A Verkhratsky $\mathrm{y}^{4,5}$ \\ Cell Death and Differentiation (2007) 14, 1235-1236; doi:10.1038/sj.cdd.4402161
}

During the last two decades, there has been a growing interest in the mechanisms that regulate cell death. The main conceptual advance, which fostered the progress in researching cell death, has been the understanding that genetically encoded programmes decide upon cell fate. ${ }^{1}$

True unicellular organisms, however, might have hardly developed specialised death programmes that would lead to their elimination. Nonetheless, the propagation and evolution based on simple cell division required extinction of unfit cells and selection of those endowed with environmental adaptation. For that reason, in initial life forms, death must have been a simple consequence of environmental injury when cell demise resulted from the inefficiency of survival mechanisms to cope with exogenous stressors. As evolution progressed, either as a result of a vertical selection or as a consequence of horizontal gene transmission, ${ }^{2,3}$ new survival pathways and protective mechanisms developed, and therefore a system of potential multiple failures instigated the appearance of several death pathways. As more altruistic functions have developed first in unicellular agglomerates ${ }^{4}$ and then in multicellular organisms, death and survival programmes began to diverge to assist cell differentiation and accomplish tissue homeostasis. The most obvious example of a primordial death mechanism would be the failure of ion homeostasis (i.e. sodium and calcium) when the harshest environmental conditions applied a tight control of ion compartmentalisation between the intra- and extracellular space. These mechanisms of cell and tissue damage are still operative today from simple organisms (e.g. Caenorhabditis elegans) to mammalian cells. In the latter, both $\mathrm{Ca}^{2+}$ and $\mathrm{Na}^{+}$overloads are one of the major causes of tissue damage upon, for example, stroke and myocardial infarction. As tissue plasticity and remodelling became a fundamental step in the evolution of complex organisms, biochemical programmes involving complex cascades leading to cell disassembly have also developed.

It is universally acknowledged that well-known death programmes, like for example, apoptosis, include biochemical cascades that are triggered by genetically controlled signalling steps. Indeed, the genetic control is probably restricted to the process of asymmetric division, which decides the fate of each and every daughter cell. The actual machinery involved in cell death execution is constitutively expressed in virtually all cells. This implies its conservation from selfish individual cells to altruistic uni- and multicellular organisms. To explain the apparent dichotomy, the death machinery as such must have been initially conserved because of its survival roles.

Imbalances in $\mathrm{Ca}^{2+}$ are the most trivial examples of death routines using physiological signalling systems. Calcium was the first to be recognised as a ubiquitous trigger of cell death in the myocardium ${ }^{5}$ later the detrimental role of disrupted $\mathrm{Ca}^{2+}$ signalling was recognised for many tissues and cell types. ${ }^{6-10}$ Importantly, deregulation of $\mathrm{Ca}^{2+}$ signalling is involved in many types of cell death: it triggers excitotoxicity in neurones and myocytes, it initiates apoptosis in many excitable and non-excitable tissues, and of course it acts as the most important activator and executor of necrotic cell death. ${ }^{11}$ All these death subroutines use existing molecular systems, which are responsible for physiological $\mathrm{Ca}^{2+}$ signalling.

Similarly, several members of the caspase family of protease, which are involved in signalling and execution of apoptosis, have physiological roles, which not only involve their proteolytic functions, but also signals mediated through their prodomains. ${ }^{12}$ Phylogenetic analyses and studies of substrate specificity indicate that human caspases can be subdivided into three different groups, one of which (caspases 1, 4 and 5) is not involved in programmed cell death, but in inflammation. This suggested that this family originated for a distinct function. This became finally evident with the identification of para-caspases and meta-caspases in lower organisms, and also by the fact that viruses learned to regulate cell death, for example via CrmA a p35, first identified by Lois Miller in 1991 in baculovirus Autographa californica. ${ }^{13}$ For a more detailed evaluation, please see the recent special issue of CDD. ${ }^{12,14-21}$ Similar considerations apply to other protease families; for example, calpains, which normally bear a variety of physiological functions, but are also involved in the promotion and progression of cell death.

The duality of survival/death signals is well exemplified by the pancreatic acinar cell, a classical model for studies of protein synthesis and secretion. Here, frequency encoded signals are essential to differentiate death or survival routines. Short-lasting, repetitive $\left[\mathrm{Ca}^{2+}\right]_{i}$ spikes - confined to the apical

${ }^{1}$ MRC Toxicology unit, Hodgkin Building, Leicester University, Lancaster Road, PO Box 138, Leicester LE1 9NH, UK; ${ }^{2}$ MRC Group, Physiological Laboratory, University of Liverpool, Crown Street, Liverpool L69 3BX, UK; ${ }^{3}$ Department of Experimental Medicine and Biochemical Sciences, University of Rome Tor Vergata, Rome, Italy; ${ }^{4}$ Faculty of Life Sciences, The University of Manchester, Manchester M13 9PT, UK and Institute of Experimental Medicine, ASCR, Videnska 1083, 142 20, Prague 4, Czech Republic

*Corresponding author: P Nicotera, MRC Toxicology unit, Hodgkin Building, Leicester University, Lancaster Road, PO Box 138, Leicester LE1 9NH, UK. Tel: + 44(0) 116252 5544; Fax: + 44161 2755463; E-mail: pn10@leicester.ac.uk 


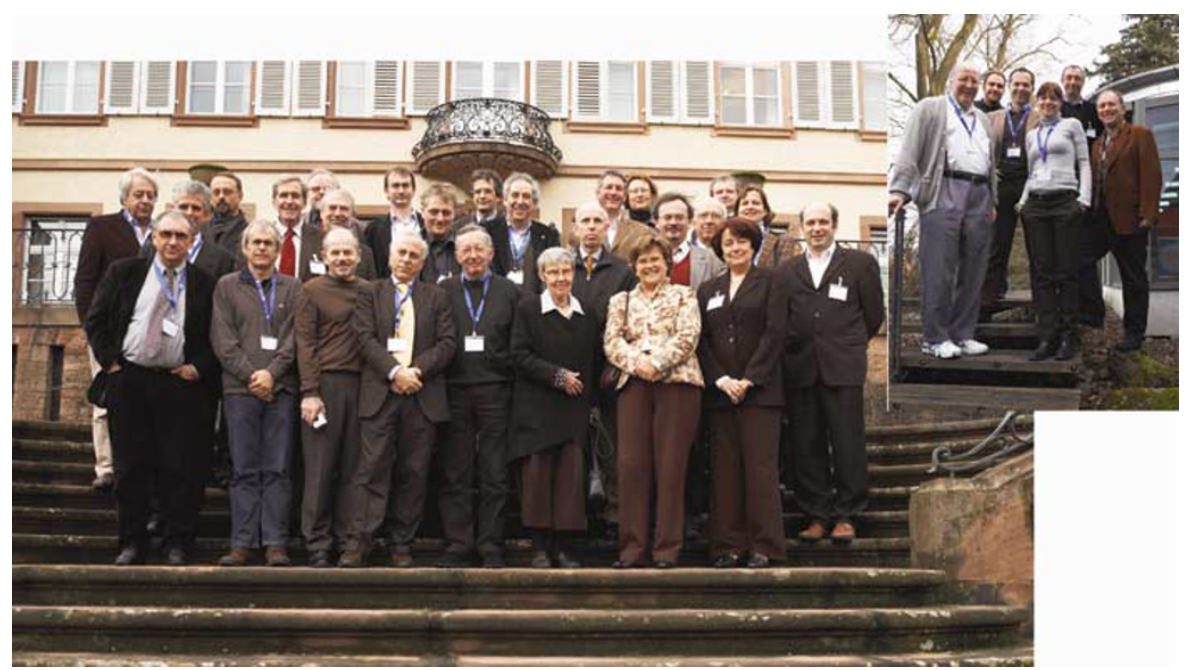

Figure 1 Photo of the participants in the meeting held at the Klaus Tschira Foundation, Heidelberg, Germany, 1-3 March 2007. Speakers and chairs: Oleg Krishtal, Carlos Matute, Pierluigi Nicotera, Theophile Godfraind, Barbro Johansson, Ana Sanchez, Eva Sykova (first row); Tullio Pozzan, Tadeush Wieloch, Sten Orrenius, Bertil Friedholm, Guido Kroemer, Ole Petersen, Alexei Verkhratsky, David Rubinsztein (second row); Lezcek Kaczmarek, Andreas Reichenbach, Frank Kirchhoff, Jean Claude Dussaule, Reimond Ardaillou, Sabine Werner (third row); Peter Krammer, Luca Scorrano, Christian Giaume, Gerry Melino (inset)

granular pole - control exocytotic secretion of digestive enzymes and ion channel opening regulating fluid secretion. ${ }^{22}$ On the other hand, prolonged global $\left[\mathrm{Ca}^{2+}\right]_{i}$ elevations induced by, for example, bile acids or alcohol metabolites cause intracellular digestive enzyme activation, vacuole formation and ultimately, necrosis, giving rise to the often fatal human disease acute pancreatitis in which the pancreas digests itself and its surroundings. ${ }^{23}$

It is remarkable that the same fundamental dualism in the survival/death signals is also utilised on a tissue and organism level. Indeed many cells, which are essential for the homeostasis of various tissues, employ the same mechanisms to ensure survival or deliver death to unfit partners. For example, glial cells, which normally act as wardens and guardians of brain homeostasis, also ensure the removal of redundant neurones. Brain insults trigger reactive gliosis, which is a well conserved defence reaction; stronger insults, however, turn reactive glia into natural killers of neurones. ${ }^{24}$ By this mechanism, glia saves the whole tissue at the expense of its injured part.

The necessity of accommodating tissue differentiation patterns has also led to diversification of death routines: while the most fundamental death mechanisms are equal in all cells, some are tissue-specific. Emergence of multiple death pathways is also evolutionarily justified, as a single and common death mechanism would have been easily hijacked by opportunistic parasitic organisms. It is, therefore, not surprising that death and life are entwined, and the understanding of death mechanisms could lead to fundamental improvement of our knowledge of survival and protection, and therefore occurrence and prevention of diseases. These guiding concepts have inspired the articles presented in this issue of Cell Death \& Differentiation. ${ }^{24-39}$

This collection of reviews, dealing with mechanisms of cells and tissue damage and repair, is based on the invited lectures given at an international conference on this broad topic. This conference, which was organized jointly by Academia Europaea and The Klaus Tschira Foundation, took place in Heidelberg, Germany in March 2007 (Figure 1).

1. Ellis HM, Horvitz HR. Cell 1986; 44: 817-829.

2. Goldenfeld N, Woese C. Nature 2007; 445: 369.

3. Vetsigian K et al. Proc Natl Acad Sci USA 2006; 103: 10696-10701.

4. Ameisen JC. Cell Death Differ 2002; 9: 367-393.

5. Fleckenstein A et al. Recent Adv Stud Cardiac Struct Metab 1974; 4: 563-580.

6. Siesjo BK. Magnesium 1989; 8: 223-237.

7. Orrenius S, Nicotera P. J Neural Transm Suppl 1994; 43: 1-11.

8. Trump BF, Berezesky IK. Faseb J 1995; 9: 219-228.

9. Choi DW. Trends Neurosci 1995; 18: 58-60.

10. Verkhratsky A. Physiol Rev 2005; 85: 201-279.

11. Orrenius S et al. Nat Rev Mol Cell Biol 2003; 4: 552-565.

12. Lamkanfi M et al. Cell Death Differ 2007; 14: 44-55.

13. Clem RJ et al. Science 1991; 254: 1388-1390.

14. Kumar S. Cell Death Differ 2006; 14: 1-2.

15. Kumar S. Cell Death Differ 2007; 14: 32-43.

16. Rozan LM, El-Deiry WS. Cell Death Differ 2007; 14: 3-9.

17. Martinon F, Tschopp J. Cell Death Differ 2007; 14: 10-22.

18. Scott AM, Saleh M. Cell Death Differ 2007; 14: 23-31.

19. Bao Q, Shi Y. Cell Death Differ 2007; 14: 56-65.

20. Timmer JC, Salvesen GS. Cell Death Differ 2007; 14: 66-72.

21. Callus BA, Vaux DL. Cell Death Differ 2007; 14: 73-78.

22. Petersen $\mathrm{OH}$. Cell Calcium 2005; 38: 171-200.

23. Petersen OH, Sutton R. Trends Pharmacol Sci 2006; 27: 113-120.

24. Giaume $C$ et al. Cell Death Differ 2007; 14: 1324-1335.

25. Galluzzi L et al. Cell Death Differ 2007; 14: 1237-1243.

26. Ott $\mathrm{M}$ et al. Cell Death Differ 2007; 14: 1243-1247.

27. Luo S, Rubinsztein DC. Cell Death Differ 2007; 14: 1247-1250.

28. Beyer TA et al. Cell Death Differ 2007; 14: 1250-1254.

29. Michaluk P, Kaczmarek L. Cell Death Differ 2007; 14: 1255-1258.

30. Sanchez A, Garcia-Sancho J. Cell Death Differ 2007; 14: 1258-1261.

31. Schapira AHV. Cell Death Differ 2007; 14: 1261-1266.

32. Giacomello M et al. Cell Death Differ 2007; 14: 1267-1274.

33. Pellegrini L, Scorrano L. Cell Death Differ 2007; 14: 1275-1287.

34. Criddle DN et al. Cell Death Differ 2007; 14: 1285-1294.

35. Prevarskaya N et al. Cell Death Differ 2007; 14: 1295-1304.

36. Nakamura T, Lipton SA. Cell Death Differ 2007; 14: 1305-1314.

37. Fredholm BB. Cell Death Differ 2007; 14: 1315-1323.

38. Sykova E, Jendelova P. Cell Death Differ 2007; 14: 1336-1342.

39. Dussaule JC, Chatziantoniou C. Cell Death Differ 2007; 14: 1343-1349. 